\title{
Proprioceptive Neuromuscular Facilitation Improves Balance and Knee Extensors Strength of Older Fallers
}

\author{
Marcelo Pinto Pereira ${ }^{1}$ and Mauro Gonçalves ${ }^{2}$ \\ ${ }^{1}$ Posture and Gait Studies Laboratory, Physical Education Department, Bioscience Institute, \\ Universidade Estadual Paulista at Rio Claro, Avenida 24A, 1515, 13506-900 Rio Claro, SP, Brazil \\ ${ }^{2}$ Biomechanics Laboratory, Physical Education Department, Bioscience Institute, Universidade Estadual Paulista at Rio Claro, \\ Avenida 24A, 1515, 13506-900 Rio Claro, SP, Brazil
}

Correspondence should be addressed to Marcelo Pinto Pereira, mppereir@yahoo.com.br

Received 7 September 2012; Accepted 4 October 2012

Academic Editors: Y. Hu, M. Syczewska, and S. P. Tokmakidis

Copyright (๑) 2012 M. P. Pereira and M. Gonçalves. This is an open access article distributed under the Creative Commons Attribution License, which permits unrestricted use, distribution, and reproduction in any medium, provided the original work is properly cited.

\begin{abstract}
Falls are one of the major problems for elderly people and proprioceptive exercises have been suggested as an alternative in rehabilitation and preventive programs. The purpose of this study was to investigate the influence of a proprioceptive neuromuscular facilitation (PNF) exercise program on balance, knee extension and flexion isometric torque, and knee extension rate of force development (RFD). Fourteen older faller subjects ( $>60$ years) were equally assigned into two groups: a control group (CG: $n=7$ ) and a training group (TG: $n=7$ ). The PNF training program was performed for 10 weeks on TG, with a frequency of three times per week. Patients were assessed before and after the PNF program, with respect to balance (Berg Balance Scale score-BBS), knee maximal isometric extension and flexion torque, knee extensor RFD, and knee extensors and flexors neuromuscular activation level and coactivation level around the knee. After 10 weeks, balance $(P<0.001)$ and knee extension torque $(P=0.05)$ were improved in TG while no differences were found for CG. These improvements were mainly attributed to central nervous system adaptations, since no differences were found for neuromuscular activation level and coactivation.
\end{abstract}

\section{Introduction}

Falls in elderly are associated to both mortality and morbidly increasing. Falls are also the major death cause in people aging over 85 years [1]. Many tools were used to discriminate people on fall risk in the past. However, the most used by its low cost is the Berg Balance Scale $[2,3]$. According to Lajoie and Gallapher [3] scores lower than or equal to 46 on Berg Balance Scale (BBS) classify subjects as fallers with $93 \%$ of specificity.

Strength reduction is pointed as the major cause of falls in this population [4] and, therefore, maximal torque should be accessed on daily-life clinical tests. The center of mass or center of pressure dislocation and velocity are useful to discriminate subjects on fall risk [5]. However, these tests require expensive equipment and high advance knowledge. This problem is not observed on isometric contractions tests, where both the strength level and the quality of power production can be observed. This latter aspect had been studied by means of rate of force development [6-10].

Lower torque and power production on elderly fallers is associated with an increased coactivation around knee and ankle $[1,11]$. Coactivation, defined as the simultaneous muscle contraction around a joint [12], had also being pointed as responsible to reduced walking speed and increase fall risk of elderly [13-15].

Beyond all exercise programs proposed to reduce fall risk in this population, proprioceptive neuromuscular facilitation (PNF) technique is presented as an interesting choice, since, according to Lee and Kerrigan [15], exercise programs with objective to enhance balance on elderly should involve coordination and proprioception activities and not only strengthen exercises $[8,16]$. During the PNF movement execution, muscles are briefly stretched before contraction, exciting neuromuscular endings which produce higher levels of force $[17,18]$. Also, the movements in PNF are executed in 
diagonal pattern that is parallel to muscular topography, which reproduces physiological movements, as gait $[17,18]$. It was also suggested that a higher balance between agonistic and antagonistic muscles activation is achieved after PNF exercises $[17,18]$ reducing coactivation. However, the effects of a PNF training program on fall risk in older people are still unknown.

The aim of this study was to evaluate the effects of a PNF exercise program on elderly on fall risk and to evaluate the neuromuscular and isometric performance response to this exercise program. As hypothesis, it is believed that both fall risk and isometric torque will be improved after training and that coactivation around knee will be reduced, since higher balance between agonistic and antagonistic muscles activation is achieved after PNF exercises.

\section{Material and Methods}

2.1. Subjects. Older community subjects were personally invited to participate in this study. They were only evaluated after signing an informed consent approved by Local Ethics Committee. As inclusion criteria, all subjects should be older than 60 years, considered as inactive-score lower or equal than 9.4 at Modified Baecke Questionnaire for older people [19] and on fall risk score of 46 or less on Berg Balance Scale [3]. Exclusion criteria were the presence of any orthopedic, cardiovascular, vestibular, psychiatric, neurological, and any other condition that would not allow the execution of all study tasks. A total of 940 subjects were invited to participate; however, only 142 subjects proposed to be evaluated. After a healthy questionnaire and inclusion and exclusion criteria, only 16 women were able to perform all the proposed tasks and met the inclusion criteria. They were randomly assigned in two groups: a training group (TG) and a control group (CG). One patient in each group failed to complete the outcome measures and both were, therefore, excluded from the study (Figure 1).

2.2. Experimental Design. Volunteers on both groups were evaluated before (pre-) and after (post-) PNF program. At the first day, after the Berg Balance Scale (BBS) application [3], volunteers answered a physical activity level and a health questionnaire. If they were eligible to participate, they returned to the laboratory on a second day (within 48 hours from the first visit). After the PNF program, data collection was not conducted more than one week apart from the last training session.

2.3. Training Program. PNF exercise program consisted of three sessions per week for a period of ten weeks, and each session was not apart from one another more than two days. Each session lasted about 90 minutes whereas eight different movement patterns (Table 1) were used-they were executed on both limbs. The same physiotherapist (appropriately instructed) conducted all training sessions where maximal manual resistance were applied; the resistance progression trough the 10 weeks was carried out according to PNF principles [20]. Also, five different training stages adapted from

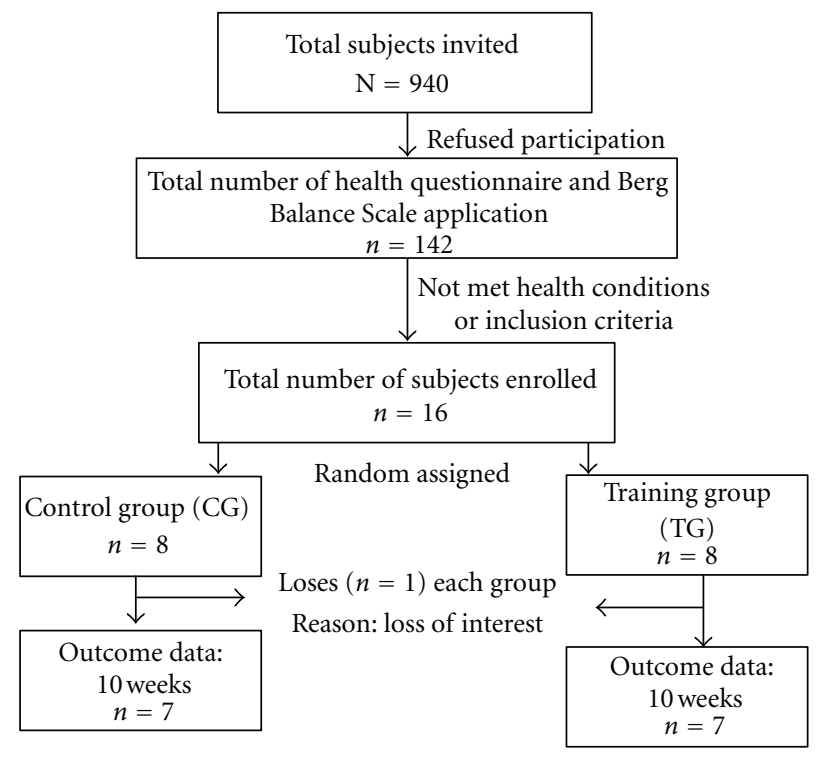

Figure 1: Study flow diagram.

Voss et al. [20] were conducted-(i) rhythmic initiation: patterns are executed in a progressive manner through passive, assisted and active movements (conducted in two initial sessions); (ii) dynamic reversion: patient moves limb isotonically through resistance following agonistic and antagonistic patterns (performed from third session to fifth training week: three series with five repetitions of each pattern, with one-minute rest interval between series and three minutes between movement patterns); (iii) isotonic combination: patient executes the agonistic movement, resist isometrically at the movement end and returns to initial position eccentrically (executed from the sixth to ninth week: two series with five repetition of each pattern, with one minute rest period between repetitions and two minutes between movements [17]; (iv) rhythmic stabilization: isometric contraction of agonist pattern followed by antagonist isometric contraction (executed on the ninth and tenth weeks, since it is the least effective technique [21]).

Stretching exercises were conducted immediately after the strengthen session on both limbs and were repeated from the second day until the last session. Contract-relax (weeks from one to five) and maintain-relax (weeks six to ten) techniques were used and all movement patterns described before were performed. Contract-relax stretch application began with the physiotherapist passively positioning the lower limb on the desired movement pattern in maximal stretching without pain (the position where an "end feel" was noted by the physiotherapist). When this position was attained, the subject was instructed to perform the antagonist movement pattern with maximal force against the physiotherapist manual restriction (a brief rotational movement was allowed). After five seconds, subject was instructed to completely relax the musculature while the physiotherapist passively moved the limb to a new maximal stretch position maintained for 15 seconds. The same procedures were adopted in maintain-relax; however, the physiotherapist did not allow 
TABLE 1: Strengthening movement patterns.

\begin{tabular}{lll}
\hline \multicolumn{2}{c}{ Initial position } & Final position \\
\hline D1-fl & $\begin{array}{l}\text { H extension, abduction, and internal rotation; K flexion; } \\
\text { A flexion and eversion; F flexion and adduction }\end{array}$ & $\begin{array}{l}\text { H flexion, adduction, and external rotation; K extension; } \\
\text { A dorsiflexion and inversion; F extension and abduction }\end{array}$ \\
D2-fl & $\begin{array}{l}\text { H extension, abduction, and internal rotation; K extension; } \\
\text { A flexion and eversion; F flexion and adduction }\end{array}$ & $\begin{array}{l}\text { H flexion, adduction, and external rotation; K flexion; } \\
\text { A dorsiflexion and inversion; F extension and abduction }\end{array}$ \\
D3-fl & $\begin{array}{l}\text { H extension, abduction, and internal rotation; K flexion; } \\
\text { A flexion and inversion; F flexion and abduction }\end{array}$ & $\begin{array}{l}\text { H flexion, adduction, and external rotation; K extension; } \\
\text { A extension and eversion; F extension and adduction }\end{array}$ \\
D4-fl & $\begin{array}{l}\text { H extension, abduction, and internal rotation; K extension; } \\
\text { A flexion and inversion; F flexion and abduction }\end{array}$ & $\begin{array}{l}\text { H flexion, adduction, and external rotation; K flexion; } \\
\text { A dorsiflexion and eversion; F extension and adduction }\end{array}$ \\
D1-ext & $\begin{array}{l}\text { H flexion, adduction, and external rotation; K flexion; } \\
\text { A dorsiflexion and inversion; F extension and abduction }\end{array}$ & $\begin{array}{l}\text { H extension, abduction, and internal rotation; K extension; } \\
\text { A flexion and eversion; F flexion and adduction }\end{array}$ \\
D2-ext & $\begin{array}{l}\text { H flexion, adduction, and external rotation; K extension; } \\
\text { A dorsiflexion and inversion; F extension and abduction }\end{array}$ & $\begin{array}{l}\text { H extension, abduction, and internal rotation; K flexion; } \\
\text { A flexion and eversion; F flexion and adduction }\end{array}$ \\
D3-ext & $\begin{array}{l}\text { H flexion, abduction, and internal rotation; } \text { K flexion; } \\
\text { A dorsiflexion and eversion; F flexion and abduction }\end{array}$ & $\begin{array}{l}\text { H extension, adduction, and external rotation; K extension; } \\
\text { A flexion and inversion; F extension and adduction }\end{array}$ \\
D4-ext & $\begin{array}{l}\text { H flexion, abduction, and internal rotation; K extension; } \\
\text { A dorsiflexion and eversion; F flexion and abduction }\end{array}$ & $\begin{array}{l}\text { H extension, adduction, and external rotation; K flexion; } \\
\text { A flexion and inversion; F extension and adduction }\end{array}$
\end{tabular}

H (hip); K (knee); A (ankle); F (feet fingers); fl (flexion); ext (extension).

any rotational movement on the contraction phase. The total stretching volume was composed by four series of 20 second for each movement pattern [22, 23].

The subjects in the CG continued their normal routine and were encouraged to maintain an active lifestyle. The examiner continued to maintain contact with the subjects during all study period.

2.4. Data Recording. Volunteers were positioned in a seated posture with hip and knees flexed at 90 degrees and ankle and spine in neutral position. A load cell (EMG System) was used to achieve the isometric knee extension and flexion torque, which were both fixed on the volunteer's right ankle and on a metal bar positioned perpendicularly to ground. After three submaximal contractions (self-selected submaximal force level) to familiarization, and three knee extension and flexion (in a random order) maximal voluntary contractions were performed, with five seconds long and five minutes rest between them. Volunteers received instructions to perform the maximal strength as quickly as possible. Force data were collected using a sample rate of $1.000 \mathrm{~Hz}$.

Circular passive bipolar $\mathrm{Ag} / \mathrm{AgCl}$ electrodes with $1 \mathrm{~cm}$ diameter of effective caption area were positioned over the vastus lateralis (VL), vastus medialis (VM), and rectus femoris (RF) and biceps femoris (BF) after shaving, abrasion and skin cleaning according to the SENIAM standard recommendations. Muscle activity was recorded at $1.000 \mathrm{~Hz}$ with a four-channel telemetric system (Telemyo 900-Noraxon) with a 10 to $500 \mathrm{~Hz}$ bandwidth and $1.000 \mathrm{x}$ gain.

2.5. Data Processing. The analyses were conducted using specific algorithms developed in MatLab. All force and electromyography variables were achieved from the trial with maximal isometric force production (maximal voluntary
contraction-MVC). Rates of force development (RFD) were determined as the slope between time and force ( $\Delta$ moment $/ \Delta$ time $)$ over time intervals of $0-0.50,0-0.10$, and $0-0.20$ seconds [10] relative to the force production onset (defined as 10\% MVC $[7,9]$ ).

The EMG root mean square was achieved from the second that presented the least variation in the load-cell data both at knee extension and flexion contractions. This one-second period was chosen by visual inspection and its location varied among the total five seconds period (i.e., in the first contraction, the one-second period chosen was 2.8-3.8 seconds and in the next contraction it was $1.5-2.5$ seconds).

Coactivation Index (CCI) was determined according to the following formula $[11,14]$ :

$$
\mathrm{CCI}=\frac{\mathrm{BF}}{\mathrm{Exe}} \times 100,
$$

where BF is the BF EMG activity (RMS) and Exe is the knee extensor (VL, RF, and VM mean) EMG activity, both obtained during knee extension contraction.

2.6. Statistical Analyze. After distribution test (ShapiroWilk test) the following procedures were conducted on the Statistica 7.0 software: to determine groups influence on baseline anthropometric and baseline data a Student- $t$ test for independent variables were used; to determine the PNF program effect on EMG, force data, and on each BBS activity and total score, paired $t$-tests were performed. A $P$ value of 0.05 was considered statistically significant.

\section{Results}

Both groups presented similar age $(72.71 \pm 6.82$ and $71.71 \pm$ 6.63 years for CG and TG), height $(1.55 \mathrm{~m} \pm 0.48$ and 
TABle 2: Mean (1SD) Berg Balance Scale (arbitrary units) by task and total for training group (TG) and control group (CG) pre- and post-training period.

\begin{tabular}{|c|c|c|c|c|}
\hline & \multicolumn{2}{|c|}{ TG } & \multicolumn{2}{|c|}{ CG } \\
\hline & Before & After & Before & After \\
\hline Sitting to standing & $3.29(0.49)$ & $3.14(0.38)$ & $3.14(0.38)$ & $3.29(0.49)$ \\
\hline Standing unsupported & $4.00(0.00)$ & $4.00(0.00)$ & $4.00(0.00)$ & $4.00(0.00)$ \\
\hline Sitting unsupported & $4.00(0.00)$ & $4.00(0.00)$ & $4.00(0.00)$ & $4.00(0.00)$ \\
\hline Standing to sitting & $2.00(0.00)$ & $3.00 *(0.82)$ & $2.43(0.53)$ & $2.29(0.49)$ \\
\hline Transfers & $3.43(0.53)$ & $3.29(0.49)$ & $3.00(0.00)$ & $3.00(0.00)$ \\
\hline Standing with eyes closed & $3.71(0.49)$ & $3.71(0.49)$ & $3.71(0.49)$ & $3.86(0.38)$ \\
\hline Standing with feet together & $3.43(0.53)$ & $4.00 *(0.00)$ & $3.71(0.49)$ & $3.86(0.38)$ \\
\hline Reaching forward with outstretched arm & $3.43(0.53)$ & $4.00 *(0.00)$ & $3.57(0.53)$ & $3.43(0.53)$ \\
\hline Retrieving object from floor & $3.71(0.49)$ & $4.00(0.00)$ & $3.86(0.38)$ & $4.00(0.00)$ \\
\hline Turning to look behind & $3.14(0.90)$ & $3.57(0.79)$ & $2.29(0.49)$ & $2.71(0.76)$ \\
\hline Turning 360 degrees & $2.71(0.76)$ & $3.57(0.79)$ & $2.71(1.11)$ & $3.57(0.53)$ \\
\hline Placing alternate foot on stool & $3.43(0.79)$ & $3.86(0.38)$ & $2.86(1.07)$ & $3.43(0.53)$ \\
\hline Standing with one foot in front & $2.14(0.38)$ & $3.29 *(0.49)$ & $2.29(1.11)$ & $2.29(1.25)$ \\
\hline Standing on one foot & $1.86(1.07)$ & $2.43(0.53)$ & $2.14(1.07)$ & $1.43(0.98)$ \\
\hline Total score & $44.29(1.60)$ & $52.71^{* *}(1.60)$ & $43.71(2.36)$ & $45.14(2.12)$ \\
\hline
\end{tabular}

${ }^{*} P<0.03$ on paired student $t$-test.

$* * P<0.01$ on paired student $t$-test.

TABLE 3: Force means (1 SD) values by group and test.

\begin{tabular}{|c|c|c|c|c|c|c|}
\hline & \multicolumn{3}{|c|}{ TG } & \multicolumn{3}{|c|}{ CG } \\
\hline & Before & After & $P$ value & Before & After & $P$ value \\
\hline \multicolumn{7}{|l|}{$\operatorname{RFD}\left(\mathrm{N} \cdot \mathrm{s}^{-1}\right)$} \\
\hline 0.50 & $527.74(291.51)$ & $526.28(249.82)$ & 0.99 & $312.01(187.02)$ & $407.47(207.47)$ & 0.39 \\
\hline 0.10 & $535.89(268.08)$ & 541.07 (219.77) & 0.96 & 343.61 (195.09) & $423.99(234.23)$ & 0.49 \\
\hline 0.20 & $477.22(171.55)$ & $491.10(165.70)$ & 0.85 & $352.22(208.91)$ & $384.68(272.23)$ & 0.74 \\
\hline \multicolumn{7}{|l|}{ Force $(N)$} \\
\hline Extension & 205.87 (47.99) & $223.55(42.59)$ & $0.05^{*}$ & $217.33(49.60)$ & $239.09(63.03)$ & 0.14 \\
\hline Flexion & $111.95(10.96)$ & $125.56(24.78)$ & 0.23 & $121.41(30.33)$ & $123.66(36.45)$ & 0.76 \\
\hline
\end{tabular}

RFD: rate of force development; TG: training group; CG: control group.

* Significant at paired student $t$-test.

$1.50 \mathrm{~m} \pm 0.64$ for CG and TG), and weight $(66.41 \mathrm{~kg} \pm 9.55$ and $61.71 \mathrm{~kg} \pm 4.55$ for CG and TG) at baseline. Also, no differences were found on the physical activity level between groups before PNF program $(6.85 \pm 4.94$ and $7.07 \pm 2.74$ for $C G$ and $T G-P=0.91$ ). No differences were also found between groups on the total BBS score at baseline; however, a significant training effect was found $(P<0.01)$ between Before and after for TG (Table 2). The only activities that were different on After in comparison to Before for TG were the "standing to sitting," "standing with feet together," "reaching forward with outstretched arm," and "standing with one foot in front" $(P<0.03)$, while no differences were observed for CG. Therefore, at After, all subjects on the TG achieved the maximal score on five of fourteen activities, while all volunteers on CG were successful to reach a perfect score just on two of fourteen activities (the same result as Before).

PNF program influenced in a positive way the maximal knee extensor torque for TG $(P=0.05)$ while no differences were found for CG (Table 3). In the same way, no differences were found on knee flexor torque for both CG and TG after the program period (Table 3 ). No differences were also seen on rate of force development (Table 2) after the ten weeks period for TG and CG $(P>0.42)$.

Figures 2 and 3 present the VL, RF, VM, and BF neuromuscular activation level during knee extension and flexion contraction, respectively. No difference were observed for any muscles on both groups during knee isometric contractions $(P>0.06)$. As seen on activation level, no differences were observed after PNF program on the coactivation index around the knee for TG (Before: $52.55 \pm 17.46 \mu \mathrm{V}$; After: $53.00 \pm 22.28 \mu \mathrm{V})$. Also, the activation level in CG has not changed (Before: $50.58 \pm 15.01 \mu \mathrm{V}$; After: $57.64 \pm 11.50 \mu \mathrm{V}$ ).

\section{Discussion}

The main result observed on results is that balance improved around $20 \%$ on training group after the PNF training, 
$\mathrm{VL}$

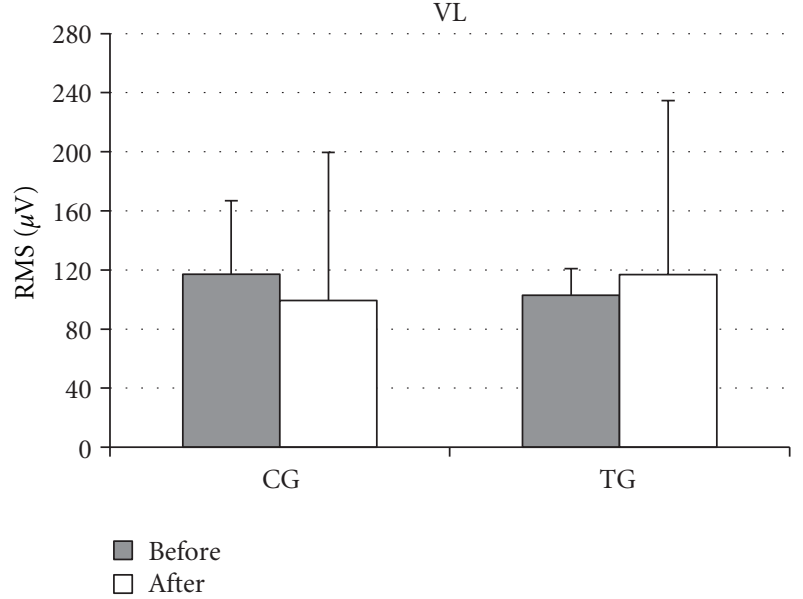

(a)

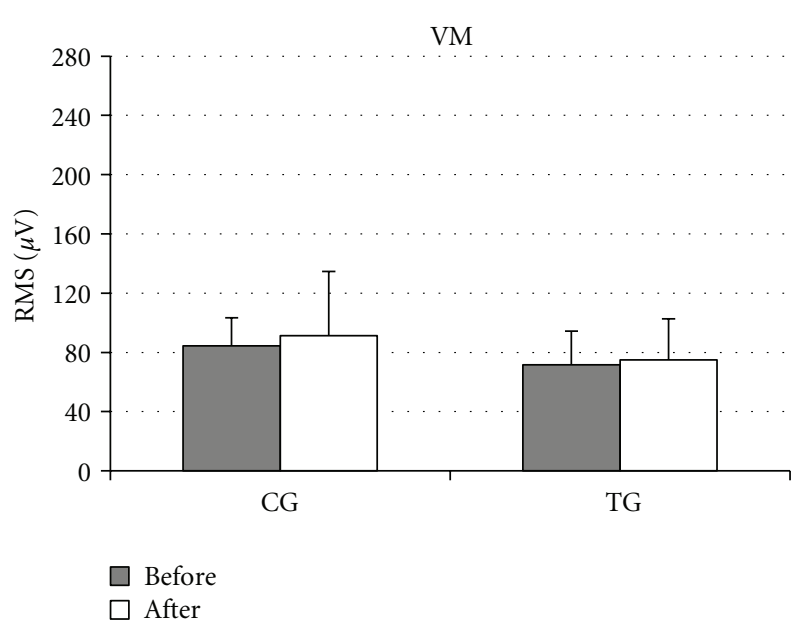

(c)

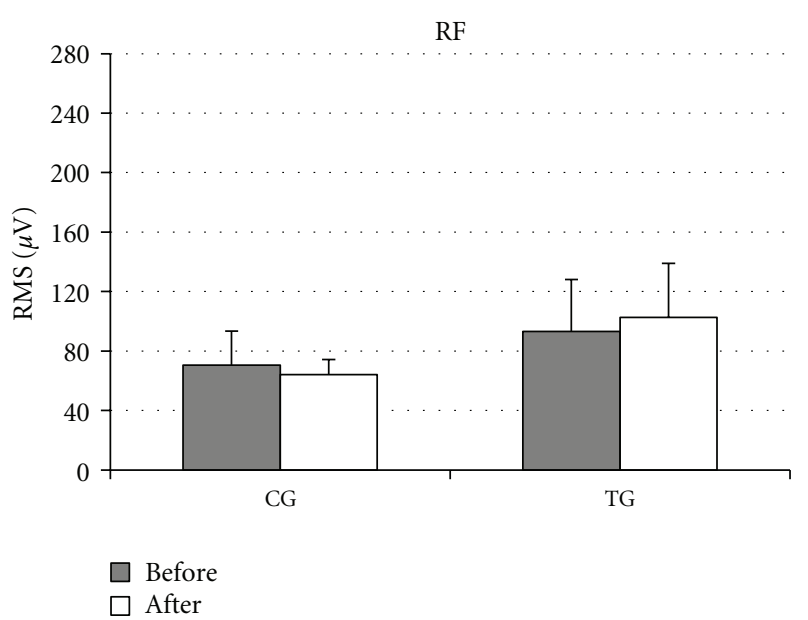

(b)

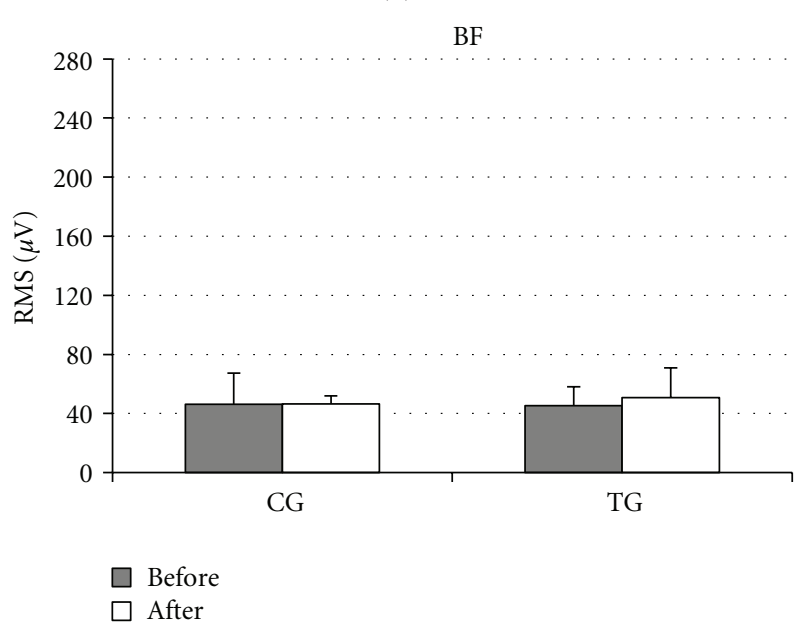

(d)

FIgURe 2: Mean (SD) knee muscles activation level during extension contraction.

while no differences were found for CG after this period. However, another interesting finding was that both rate of force development and neuromuscular variables were not influenced by this program. Some explanations could be attributed to this lack of response to PNF program on these variables. (i) PNF does not result in adaptations on muscles fibers adaptations, as already found by others [17]. Hence, since rate of force development is direct related to muscle fibers composition [10] this result was not unexpected. (ii) the effect of training programs on rate of force development was attributed to neuromuscular adaptations, as the increase in the motor neuron recruitment level [10]. Since we found no EMG differences between before and after 10 weeks (Figures 2 and 3), this could explain our lack of results on RFD. This data is also in agreement with other studies, which have shown that elderly do not increase their RFD after strengthening training [8].

The lack of neuromuscular response to PNF program could be first attributed to the idea that test condition (isometric contraction on single plain) was not similar to exercises patterns (diagonal movements) and, therefore, results could not be observed due to training type specificity [24]. It could be speculated that pre- and post-evaluations should be performed on the same exercises movement patterns. However, the intention of this study was to simulate evaluations normally conducted on clinical day-life and these involve strength tests performed on single movement planes.

Beside the lack of neuromuscular variables response, knee extension strength was positively affected by PNF program. Coactivation reduction could be responsible to explain a strength increase without neuromuscular response [25]. However, as coactivation was also not influenced by PNF program (Table 3 and Figure 3), this last theory is unacceptable. According to Jesper [26], in some conditions, strength improvement can be attributed to supraspinal neural adaptations and not to peripheral paths, as normally seen. Therefore, since PNF exercises enhance proprioceptive response [17] and therefore promotes higher neural excitement level on cortex [27], the training characteristics seem to be responsible to explain the strength improvements observed on this study. However, this is only an assumption, since no evidences were investigated on this study to properly affirm 


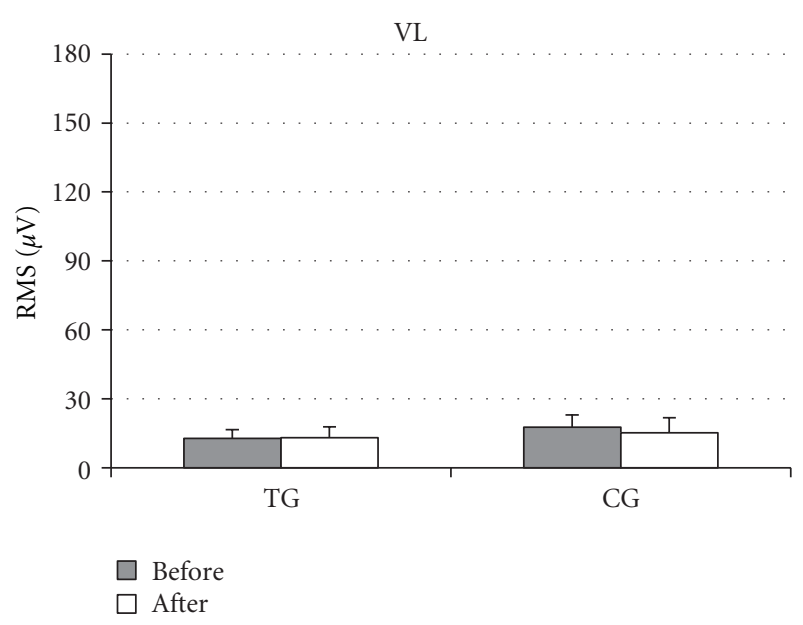

(a)

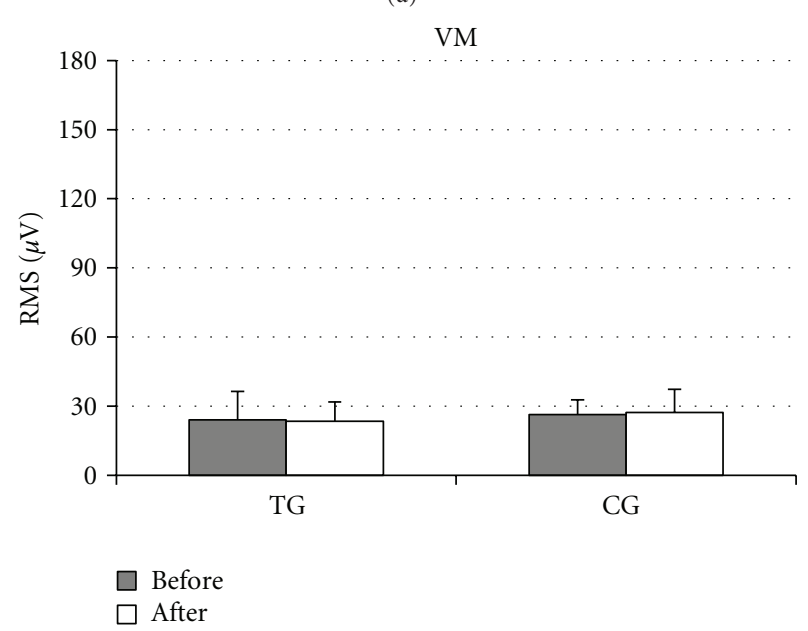

(c)

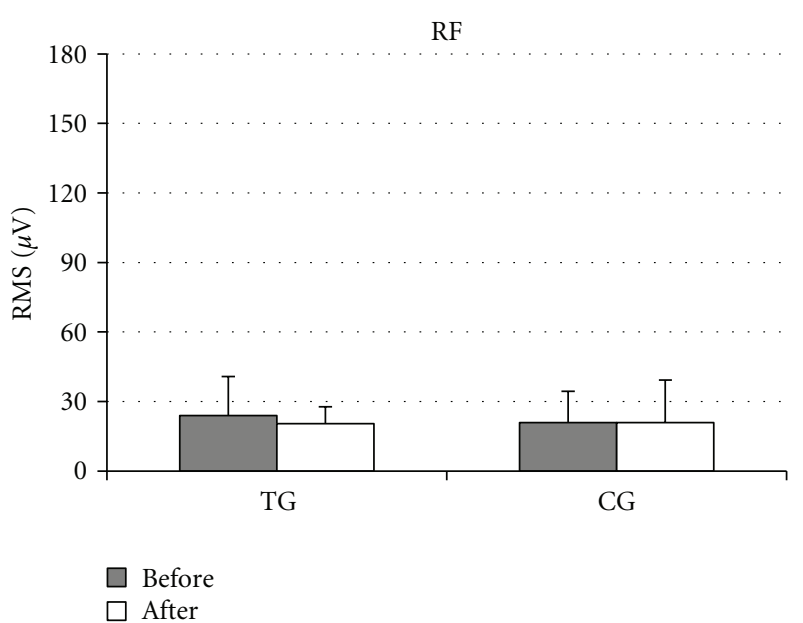

(b)

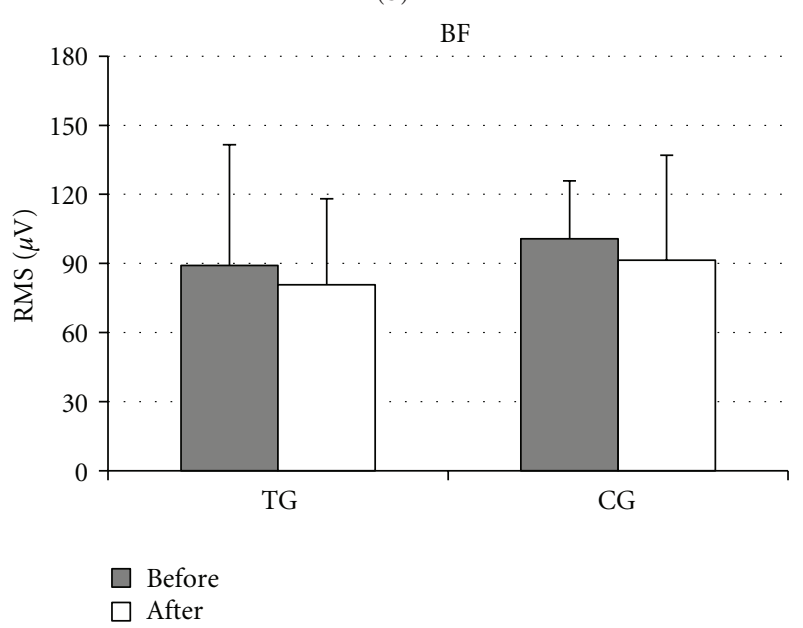

(d)

FIGURE 3: Mean (SD) knee muscles activation level during flexion contraction.

that. Another explanation, obviously more plausible, is that our training program improved proprioception, which could explain both the improvement on balance and knee extension strength without neuromuscular response. However, we failed to investigate directly proprioceptive variables as the position sense.

However, our results let us to affirm that exercise programs involving limb movements which follow diagonal movement patterns, parallel to muscular topography and similar to that used on day-life activities can prepare subjects to respond in a more properly way to balance [26] disturbances. We can affirm that, since we found higher Berg Balance Scale scores on TG, without response on CG. This result is highly important to elderly, since they are more prone to falls episodes that can lead to an increase in mortality and financial expenses.

Hence, it is also suitable to affirm that PNF training program used in this study induce a response more related to an appropriate reaction to balance perturbation than to strength level improve. This is confirmed when PNF training response to individual tasks on Berg Balance Scale were accessed (Table 2). A higher performance after training was achieved on activities where balance were perturbed, as standing with feet together, standing with one foot in front and reaching forward with outstretched arm, both statistically different when comparing Before versus After (Table 2). However, when tasks that involve strength were analyzed, as sitting to standing or placing alternate foot on stool, no differences were found between Before versus After. Therefore, it is suggested that in future studies, the effect of PNF should be tested on dynamic conditions as gait. This is a clear study limitation. Also, the low number of subjects investigated in this study should be considered as a study limitation. We could not discard the Hawthorne effect and also it is not possible to affirm properly that PNF implies in higher effects than other exercises methods, since no comparisons were conducted. However, this was not the objective of this study and therefore it is suggested that future investigations consider this topic.

An expressive improvement on fall risk in elderly after a proprioceptive neuromuscular facilitation program were seen in the present study, since volunteers presented a higher Berg Balance Scale performance and a higher knee extensor strength level after training. Also, proprioceptive integration 
or others central modulation processes and not peripheral adaptations are suggested to be responsible to such results, since no training effect were seen on both electromyographic variables and knee extensor rate of force development.

\section{Acknowledgment}

This paper is supported by Fundação de Amparo a Pesquisa do Estado de São Paulo: FAPESP.

\section{References}

[1] H. J. Lee and L. S. Chou, "Detection of gait instability using the center of mass and center of pressure inclination angles," Archives of Physical Medicine and Rehabilitation, vol. 87, no. 4, pp. 569-575, 2006.

[2] K. O. Berg, S. L. Wood-Dauphinee, J. I. Williams, and B. Maki, "Measuring balance in the elderly: validation of an instrument," Canadian Journal of Public Health, vol. 83, no. 2, supplement, pp. S7-S11, 1992.

[3] Y. Lajoie and S. P. Gallagher, "Predicting falls within the elderly community: comparison of postural sway, reaction time, the Berg balance scale and the Activities-specific Balance Confidence (ABC) scale for comparing fallers and non-fallers," Archives of Gerontology and Geriatrics, vol. 38, no. 1, pp. 11-26, 2004.

[4] M. Pijnappels, J. C. E. van der Burg, N. D. Reeves, and J. H. van Dieën, "Identification of elderly fallers by muscle strength measures," European Journal of Applied Physiology, vol. 102, no. 5, pp. 585-592, 2008.

[5] I. Melzer, N. Benjuya, and J. Kaplanski, "Postural stability in the elderly: a comparison between fallers and non-fallers," Age and Ageing, vol. 33, no. 6, pp. 602-607, 2004.

[6] C. Suetta, P. Aagaard, S. P. Magnusson et al., "Muscle size, neuromuscular activation, and rapid force characteristics in elderly men and women: effects of unilateral long-term disuse due to hip-osteoarthritis," Journal of Applied Physiology, vol. 102, no. 3, pp. 942-948, 2007.

[7] J. W. Bellew, "A Correlation analysis between rate of force development of quadriceps and postural sway in healthy older adults," Journal of Geriatric Physical Therapy, vol. 1, pp. 1-6, 2002.

[8] J. W. Bellew, J. W. Yates, D. R. Gater, and J. L. Clasey, "Explosive force production in older adults following a traditional strength training program," Journal of Geriatric Physical Ther$a p y$, vol. 26, pp. 9-13, 2003.

[9] S. H. J. Chang, V. S. Mercer, C. A. Giuliani, and P. D. Sloane, "Relationship between hip abductor rate of force development and mediolateral stability in older adults," Archives of Physical Medicine and Rehabilitation, vol. 86, no. 9, pp. 1843-1850, 2005.

[10] P. Aagaard, E. B. Simonsen, J. L. Andersen, P. Magnusson, and P. Dyhre, "Increased rate of force development and neural drive of human skeletal muscle following resistance training," Journal of Applied Physiology, vol. 93, no. 4, pp. 1318-1326, 2002.

[11] N. D. Reeves, C. N. Maganaris, and M. V. Narici, "Plasticity of dynamic muscle performance with strength training in elderly humans," Muscle and Nerve, vol. 31, no. 3, pp. 355-364, 2005.

[12] K. Falconer and D. A. Winter, "Quantitative assessment of cocontraction at the ankle joint in walking," Electromyography and Clinical Neurophysiology, vol. 25, no. 2-3, pp. 135-149, 1985.

[13] M. P. Pereira and M. Goncalves, "Effects of fatigue induced by prolonged gait when walking on the elderly," Human Movement, vol. 12, no. 3, pp. 242-247, 2011.

[14] M. P. Pereira and M. Gonçalves, "Muscular coactivation (CA) around the knee reduces power production in elderly women," Archives of Gerontology and Geriatrics, vol. 52, no. 3, pp. 317$321,2011$.

[15] L. W. Lee and D. C. Kerrigan, "Identification of kinetic differences between fallers and nonfallers in the elderly," American Journal of Physical Medicine and Rehabilitation, vol. 78, no. 3, pp. 242-246, 1999.

[16] K. Häkkinen, A. Pakarinen, W. J. Kraemer, A. Häkkinen, H. Valkeinen, and M. Alen, "Selective muscle hypertrophy, changes in EMG and force, and serum hormones during strength training in older women," Journal of Applied Physiology, vol. 91, no. 2, pp. 569-580, 2001.

[17] N. Kofotolis and E. Kellis, "Effects of two 4-week proprioceptive neuromuscular facilitation programs on muscle endurance, flexibility, and functional performance in women with chronic low back pain," Physical Therapy, vol. 86, no. 7, pp. 1001-1012, 2006.

[18] M. P. Pereira, "Proprioceptive neuromuscular facilitation does not increase blood pressure of healthy elderly women," Physiotherapy Theory and Practice, vol. 28, no. 5, pp. 412-416, 2012.

[19] L. E. Voorrips, A. C. Ravelli, P. C. Dongelmans, P. Deurenberg, and W. A. Van Staveren, "A physical activity questionnaire for the elderly," Medicine and Science in Sports and Exercise, vol. 23, no. 8, pp. 974-979, 1991.

[20] D. E. Voss, M. K. S. Ionta, and B. J. Myers, Proprioceptive Neuromuscular Facilitation, Harper \& Row, New York, NY, USA, 1985.

[21] K. Hazaki, N. Ichihashi, and T. Morinaga, "Electromyographic analysis of thigh muscles in pnf patterns of the lower extremity: muscle activities in the lengthened range," Journal of Physical Therapy Science, vol. 8, no. 1, pp. 29-32, 1996.

[22] W. L. Cornelius and M. R. Rauschuber, "The relationship between isometric contraction duration and improvement in acute hip joint flexibility," Journal of Strength and Conditioning Research, vol. 1, pp. 39-41, 1987.

[23] T. Hortobagyi, J. Faludi, J. Tihanyi, and B. Merkely, "Effects intense "stretching"-flexibility training on the mechanical profile of the knee extensors and on the range of motion of the hip joint," International Journal of Sports Medicine, vol. 6, no. 6, pp. 317-321, 1985.

[24] W. J. Kraemer and N. A. Ratamess, "Fundamentals of resistance training: progression and exercise prescription," Medicine and Science in Sports and Exercise, vol. 36, no. 4, pp. 674-688, 2004.

[25] A. Ferri, G. Scaglioni, M. Pousson, P. Capodaglio, J. Van Hoecke, and M. V. Narici, "Strength and power changes of the human plantar flexors and knee extensors in response to resistance training in old age," Acta Physiologica Scandinavica, vol. 177, no. 1, pp. 69-78, 2003.

[26] L. Jesper, "Central nervous adaptations to strength training," in Proceedings of the 12th Annual Congress of the ECSS, Jyväskylä, Finland, 2007.

[27] K. Shimura and T. Kasai, "Effects of proprioceptive neuromuscular facilitation on the initiation of voluntary movement and motor evoked potentials in upper limb muscles," Human Movement Science, vol. 21, no. 1, pp. 101-113, 2002. 


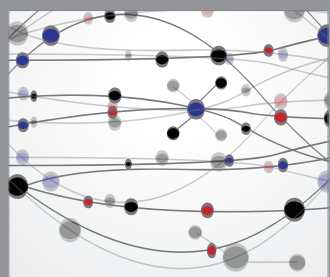

The Scientific World Journal
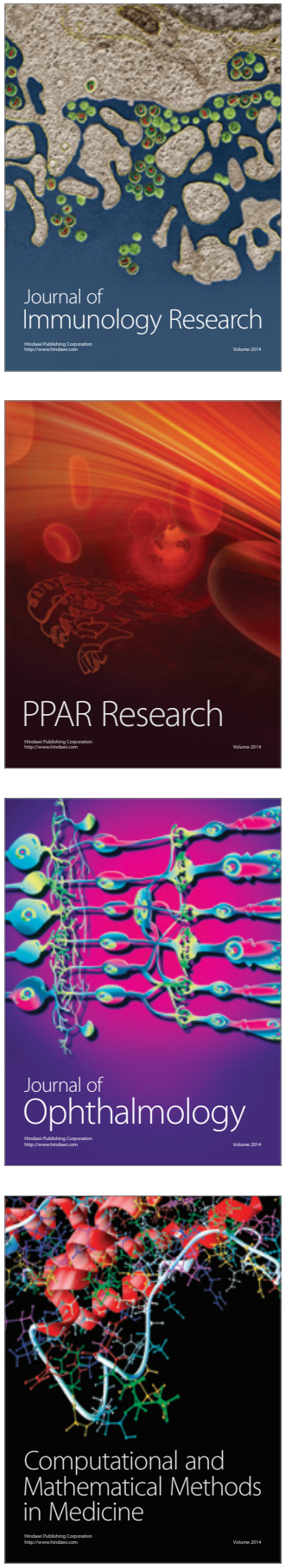

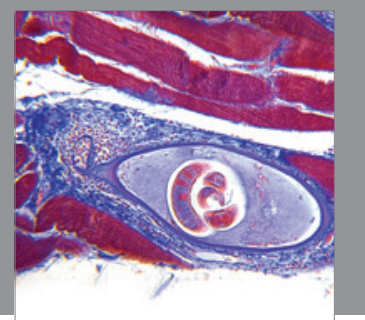

Gastroenterology

Research and Practice
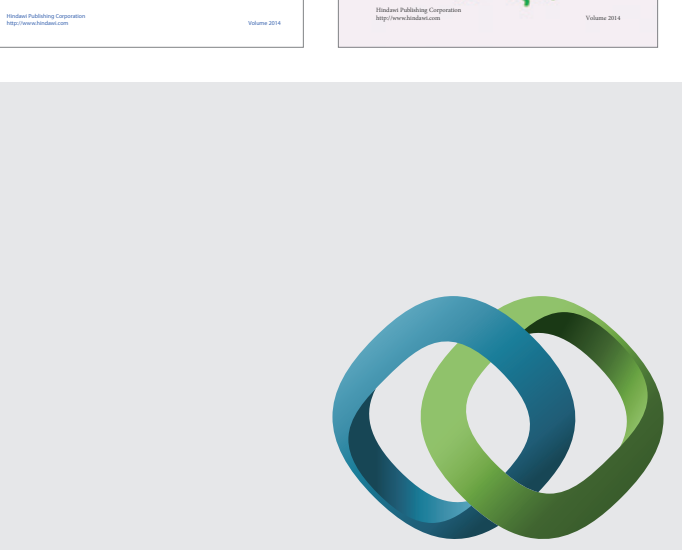

\section{Hindawi}

Submit your manuscripts at

http://www.hindawi.com
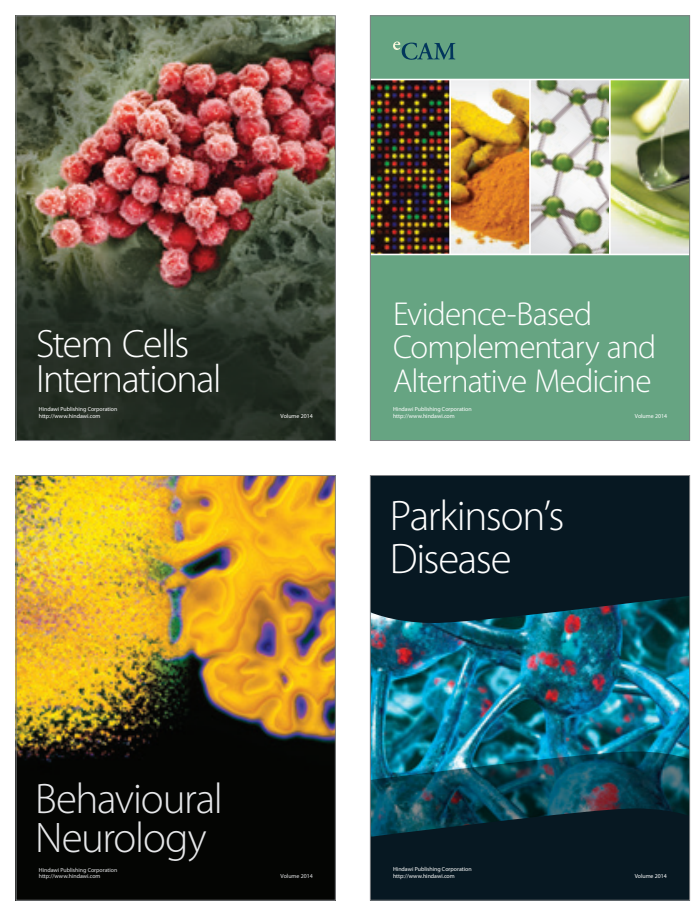

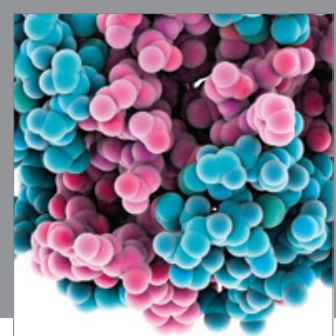

Journal of
Diabetes Research

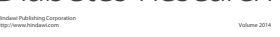

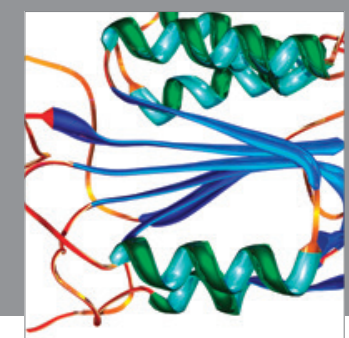

Disease Markers
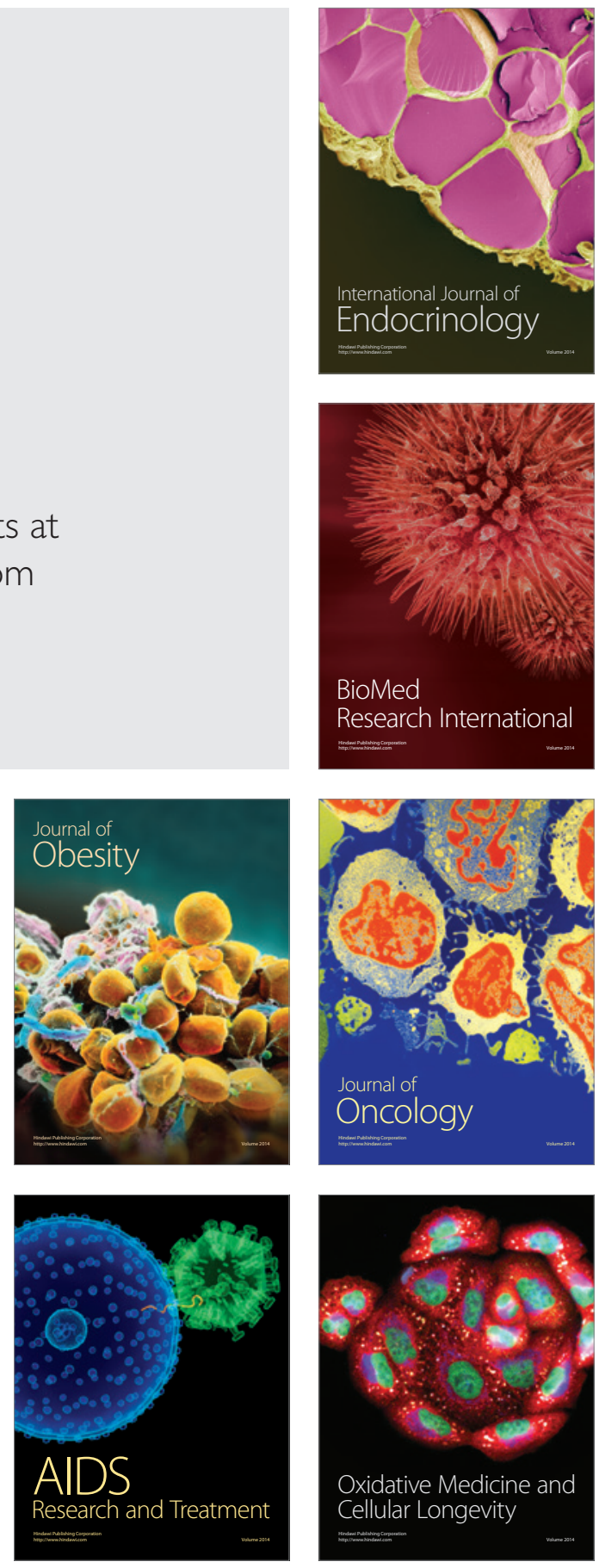